\title{
Localized Aspergillus Thyroiditis In Patients With Diabetic Nephropathy
}

\author{
Elif Şahin Horasan, Ramazan Gen*, Esen Akbay*, Yusuf Vayısoglu**, Ahmet Kıykım* \\ Mersin University Medical Faculty, Department of Infection Diseases, MERSİN, TURKEY \\ *Department of Endocrinology and Metabolic Diseases, MERSIN, TURKEY \\ **Department of Otorhinolaryngology, MERSİN, TURKEY \\ $\star \star \star$ Department of Nephrology, MERSİN, TURKEY
}

\section{INTRODUCTION}

Aspergillosis is increasingly recognized as an important oppurtunistic pathogen in immunocompromised patients. Fungal infections of the thyroid are uncommon. Previous autopsy studies revelead that $9-15 \%$ of disseminate invasive aspergillosis involves the thyroid. Most patients with thyroidal invasion remain asymptomatic and it is usually difficult to diagnose in the antemortem period. The underlying conditions of Aspergillosis included hematological malignancies, organ transplantation, and autoimmune diseases treated with corticosteroids and cytotoxic drugs.

To our knowledge there was no reports in the literature about the aspergillus thyroiditis in dialysis patients. In this presentation, we demonstrated relief of symptoms and improvment of the aspergillus thyroiditis in patient with chronic renal failure (CRF) due to diabetic nephropathy who was treated with voriconazole

CASE REPORT

A 65-year-old man, who had been on hemodialysis for 18 monts due to renal failure secondary to diabetic nephropathy was admitted to our nephrology department due to disseminated athralgy and malaise. He was scheduled to receive three sessiond of hourly HD per week. Patient had a history of diabetes mellitus for more than 10 years and used insulin regularly. On admission, vital signs were stable. There was not pathologic findings on physical examinations. On admission, blood leukocyte count was $5800 / \mathrm{mL}$, erythrocyte sedimentation rate (ESR) was $62 \mathrm{~mm} /$ hour, procalsitonin was $0.4 \mathrm{ng} / \mathrm{mL}$. and C-reactive protein (CRP) was $332 \mathrm{mg} / \mathrm{L}$ (normal level 0-5mg/L). Rutine blood, urine and sputulum cultures of pateints were negative. During the following period, patient had no fever. Chest X-ray showed no abnormality. Computed tomographic scan of all body was taken for investigation of elaveted CRP and ESR. Cranial, abdominal and pelvic tomography revealed no abnormality. Chest thomography revealed left thyroid lobe enlargment with nearly $3 \times 3 \mathrm{~cm}$ hypodense nodular lesion in the left thyroid lobe.

Thyroid ultrasonogrpahy (USG) was taken for investigate the thyroid. Thyroid USG revealed; right lobe size was $42 \times 22 \times 48 \mathrm{~mm}$, left lobe size was $45 \times 26 \times 54 \mathrm{~mm}$ and isthmus size was $7 \mathrm{~mm}$ and nonencapsulated, mix-echoic $(70 \%$ solid, $30 \%$ cystic) nodule $38 \times 25 \mathrm{~mm}$ in size with microcalcification at the left lobe (figure). Thyroid function test was normal. A fine needle aspiration biopsy was performed to determine the aetiology of thyroid nodul. Cytologically, there was benign thyroid epithelium with inflammatory debris as well as filamentous organisms with septate hyphae and acute angle branching (45 degrees), consistent with Aspergills infection. High resolution computed tomograghy (HRCT) of the chest was taken for investigation of aspergillosis focus. HRCT of the chest showed no evidence of intrapulmonary fungal lesions. Cultures of the sputulum and thyroid aspiration were negative for aspergillus. We started voriconazole treatment. After treatment with voriconazole, CRP was reduced from $332 \mathrm{mg} / \mathrm{L}$ to $5 \mathrm{mg} / \mathrm{L}$, ESR was reduced from $62 \mathrm{~mm} /$ hour to $11 \mathrm{~mm} / \mathrm{hour}$ and symptoms was relified.

\section{CONCLUSION}

In conclusion, localized Aspergillus thyroiditis is an uncommon presentation of aspergillosis. Many risk factors and conditions have been described for the development of disease, but there are no reports in patient with renal failure secondary to diabetic nephropathy. A high index of suspicion is necessary for early diagnosis and treatment, which are essential to good outcome. 